\title{
Performance of Channel Prediction Using 3D Ray-tracing Scheme Compared to Conventional 2D Scheme
}

\author{
Nam-Ryul Jeon, Chang-Hoon Lee, Noh-Gyoung Kang, and Seong-Cheol Kim \\ Institute of New Media and Communications, Seoul National University, \\ Shilim-dong, Kwanak-gu, Seoul, Republic of Korea, 151-742
}

\begin{abstract}
Channel prediction tools using ray-tracing scheme can be used to get information of communication channel instead of channel measurement. Three dimensional (3D) ray-tracing technique is known for the better accuracy compared to two dimensional (2D) ray-tracing. But it requires a lot of calculations, thus 2D simulation has been generally performed. This paper shows how precious 3D ray-tracing predicts the received power indoors compared to 2D one. For this work, wave propagation phenomena are analyzed using Finite Difference Time Domain (FDTD) method and the channel measurement data also will be offered as a standard of comparison.
\end{abstract}

Index terms - ray-tracing, 3D, channel measurement, simulation, FDTD, indoor, channel modeling.

\section{I.INTRODUCTION}

A Wireless Local Area Network (WLAN) system does not always operate with its best performance. The data rate on the system can decrease below what was expected or the communication link can be totally broken in many actual situations. There are many adverse conditions which may deteriorate the performance of WLAN systems. Particularly in physical layer, the following situations can be counted. First, we can consider the situation that the transmitted power from an Access Point (AP) do not spread over all the target area enough to detect the transmitted signal. Secondly, it can be due to the strong frequency selectiveness in a multi-path channel. Badly distorted by such a strong selectiveness in frequency domain, the transmitted signal cannot be recovered at the receiver even being used equalizers or channel code-decode techniques. And last, the unwanted signals from other APs or other kind of devices can interfere with receiving the wanted signal. To overcome above situations, first of all, we need to obtain the channel information as the channel impulse response of the target links. They may think it is necessary to collect the channel information from a number of channel measurement. Actually, we have usually got it from channel measurements in various indoor environments till now. However, it will be too time consuming to measure all the channels over the WLAN service area. Besides, the channel measurement system has limited resolution in itself and the observational errors which are made during measurement procedure may not be ignorable [1].

Channel prediction tools using ray-tracing technique can be used as an alternative solution. They give the channel information predicted from the blueprint of the target environment using geometrical optics simulation without wasting time and labor. 2D Ray-tracing schemes are already well-known as good channel prediction techniques in [2], [3]. Recently three dimension (3D) ray-tracing techniques have been studied as in [4], [5]. Generally, it is known that the prediction performance using 3D ray-tracing scheme is better than 2D one. However, the reasonable comparison of prediction performance between 2D and 3D was not studied sufficiently. In this paper, we will give the channel prediction performance using 3D ray tracing compared with 2D. And the channel measurement data are also given as a standard of channel prediction performance.

This paper is organized as follows. Section II gives a brief introduction about wave propagation modeling in 2D. In Section III, wave propagation phenomena in 3D are analyzed using FDTD method. Section IV shows how 3D ray-tracing scheme can be derived from 2D one. In Section $\mathrm{V}$, the performance of $3 \mathrm{D}$ ray-tracing is compared to 2D ray-tracing and channel measurement data. Finally, Section VI presents the conclusions of this study.

\section{WAVE PRopagation ModeLING IN 2D}

Assume that $P_{t}$ is the total transmitted power, $G_{t}\left(\phi_{t}\right)$ and $G_{r}\left(\phi_{r}\right)$ are the transmit antenna gain at the transmitted angle $\phi_{t}$ and the receive antenna gain at the received angle $\phi_{r}$, respectively. Let a transmitted wave whose wave length is $\lambda$ propagate from a transmit antenna to a receive antenna after $n$ times reflections or 
transmissions at walls, the total unfolded distance of the propagating path is $d$, the Fresnel reflection coefficient and transmission coefficient of walls at which the wave arrives $i$-th are represented as $\Gamma_{i}, \tau_{i}$, respectively. $I_{i}$ is the interaction coefficient which has the unified concept of the reflection coefficient and transmission coefficient. Then a propagation model can be described by the following received power of a path $P^{2 D}(d)$ :

$$
P^{2 D}(d)=\frac{P_{t} \lambda^{2}}{(4 \pi)^{2}} \frac{1}{d^{2}} G_{T}\left(\phi_{t}\right) G_{R}\left(\phi_{r}\right)\left(\prod_{i=0}^{n} I_{i}\left(\phi_{i}\right)\right)^{2} .
$$

The above model is used in $2 \mathrm{D}$ ray-tracing simulation.

\section{WAVE PROPAGATION IN 3D}

The primary difference between 3D ray-tracing and 2D one is whether wave propagation phenomena at ceilings and floors are considered. This section shows how much propagations at ceilings and floors affect the total received power, and also gives the method to obtain Fresnel coefficients at ceilings and floors using FDTD method.

\section{PROPAGATION AT CEILING AND FlOOR}

Generally, floors and ceilings consist of thick concrete layer and a latticed metal frame is inserted into the middle of the concrete layer for the strength of the building.

Due to the latticed frame of the ceilings or the floors, they can not be modeled as multi-layered panels which can be characterized using boundary conditions easily. For making a special model for these wall structures, we introduced (FDTD) scheme, which can give the information about spreading patterns of the transmitted waves' power in an environment with a relatively small dimension.

The scenario for this FDTD simulation is as shown in figure 1 . The thickness of concrete layer is $30 \mathrm{~cm}$, whose permeability is 6.25-j0.37. The metal frames inserted at the middle of ceilings and floors form the lattice whose interval is $10 \mathrm{~cm}$. A dipole antenna is used as a transmit antenna. And the target frequency is $5.8 \mathrm{GHz}$ used in 802.11a standard, the total power radiated from the transmit antenna is assumed to be 1 . The simulation algorithm is programmed not to receive wave components which are propagated through direct path from transmitter to receiving point. To see the effects of transmissions one group of observation points is located above the ceiling. The other group is below the ceiling for reflections. This scenario may be regarded as what to see the effects at only ceiling. But the effects at floor can be deduced from effects of ceiling reciprocally.

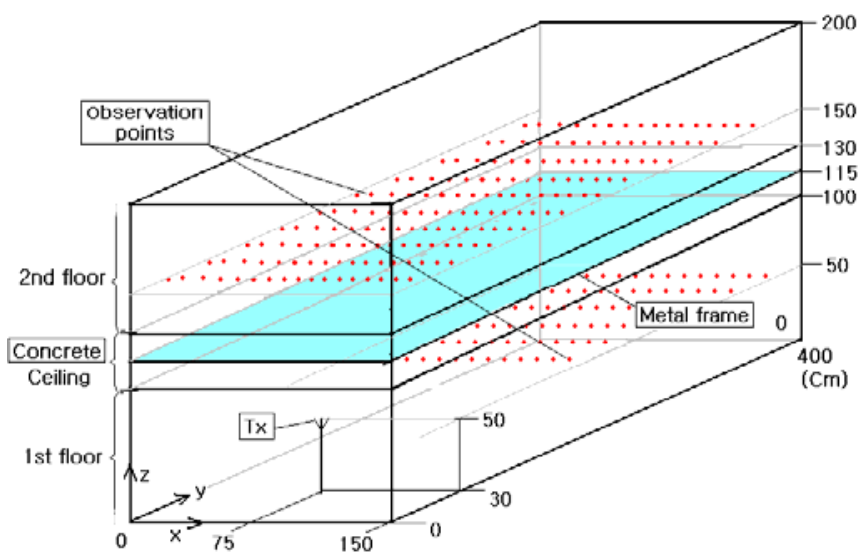

Figure 1. The overall figure of the scenario for FDTD simulation

\section{1) Transmission at Ceiling and Floor}

The powers at points in height of $150 \mathrm{~cm}$ are depicted in figure 2. We can see that the minimum power loss after penetrating the ceiling is larger than $67 \mathrm{~dB} .67 \mathrm{~dB}$ attenuated component itself is large enough. Moreover, many metallic fixtures such as water pipes, power lines, lightening fixtures exist inside ceiling structures and these interrupt the wave penetrating a ceiling additionally. After considering all these factors, it is reasonable to neglect the transmissions at ceilings and floors.

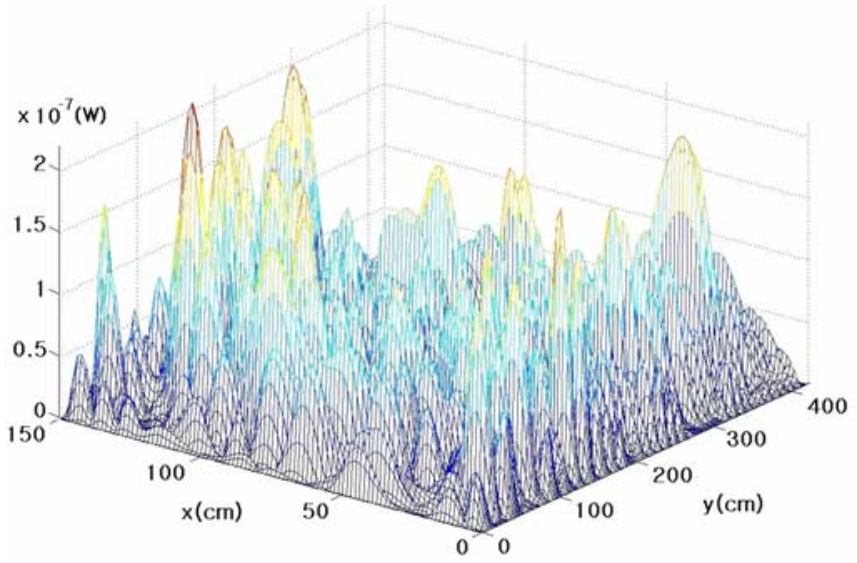

Figure 2. The received power in the height $\mathrm{z}=150 \mathrm{~cm}$

\section{2) Reflection at Ceiling and Floor}

In the figure 3 , the powers at all the points which are in the same height as the transmit antenna are depicted.

As shown in the figure, the received power is not negligible compared to the transmit power. We can find that the power of the components reflected at the ceiling 
or floor is an important factor affecting the total received power, which makes the differences of the prediction performance between 2D and 3D ray-tracing.

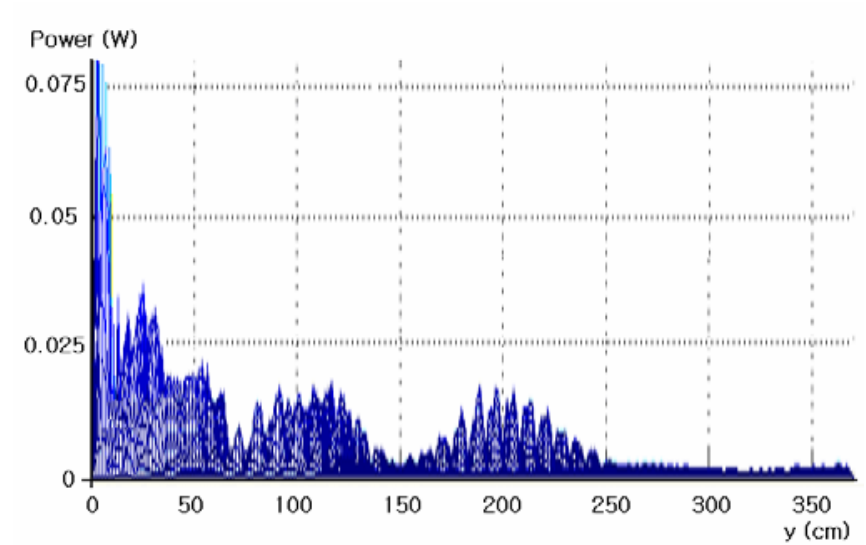

Figure 3 . The received powers in the height $\mathrm{z}=50 \mathrm{~cm}$

\section{FRESNEL COEFFICIENTS AT CEILING AND FLOOR}

The reflection coefficients of the ceilings and floors can be derived from the results of FDTD simulations.

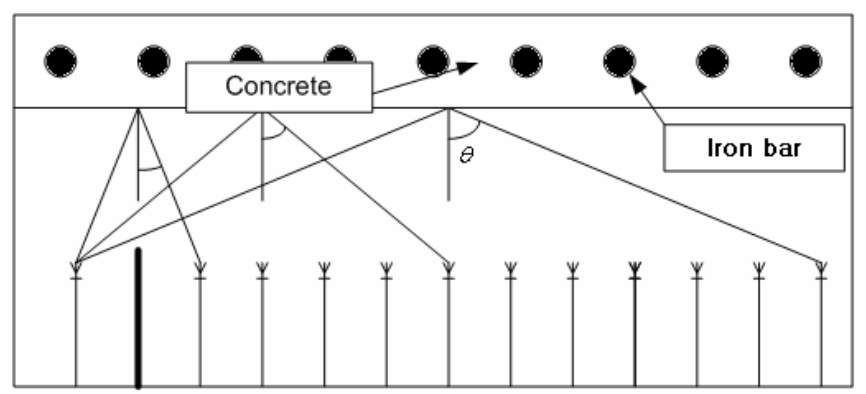

Figure 4. The model for deriving Fresnel coefficients at ceilings and floors from FDTD simulations.

For receive points as shown in figure 4, we can regard the computed electric field strengths and phases for the incident angle of $\theta$ as the reflection coefficient of the ceiling. After normalizing with respect to each distance and antenna gain, the Fresnel reflection coefficient is obtained. The amplitude of reflection coefficient from the FDTD simulation results are depicted in figure 5.

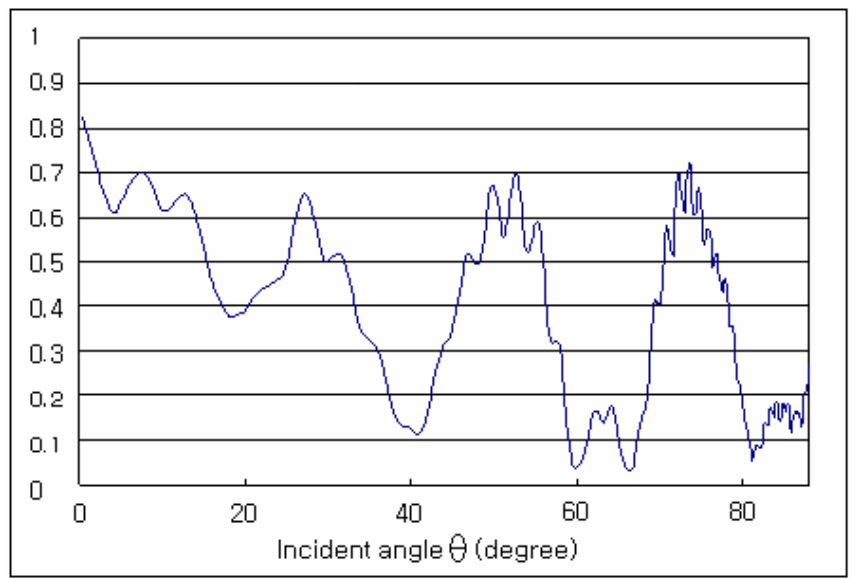

Figure 5. The amplitude of Fresnel reflection coefficient obtained from FDTD simulation

\section{Simple 3D WAVE PRopagation Model}

The propagating paths in 3D can be deduced from the paths in 2D [6]. As shown in Figure 6, considering the reflections at the ceiling and floor, additional paths is derived from a 2D path. This method can be applied to the 2D paths which include one or more reflections or transmissions at vertical walls. Theoretically, the infinite number of 3D paths can be increased in this way. However, in practice, we have to consider followings.

a. The dipole antenna commonly used as a transmitter and a receiver has a serous gain at the direction having large elevation angle offset from the main beam direction.

b. Generally, the received power is small when the number of reflections is large.

c. The longer the total propagation path, the weaker the received power.

These facts inform that finding only a few significant $3 \mathrm{D}$ paths from a 2D path is enough to improve the prediction accuracy. In this work, we considered only seven 3D paths per a 2D path as shown in Figure 6.

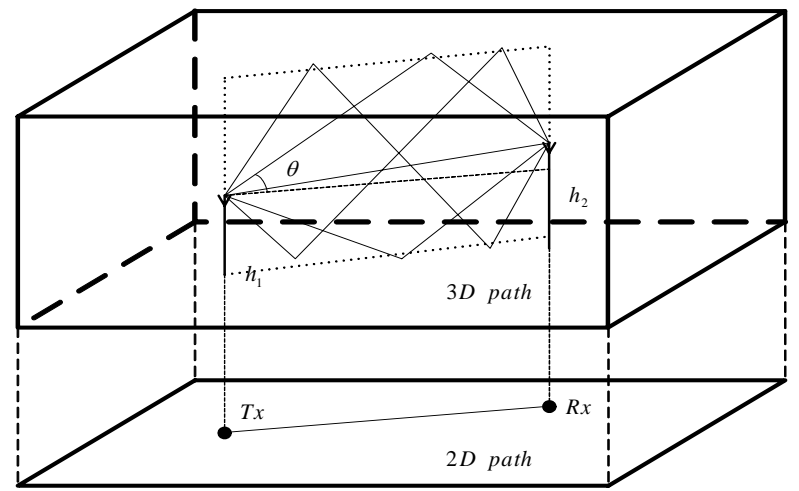

Figure 6 . The 3D paths derived from a 2D path 
The parameters used in 3D modeling are summarized in table 1.

\begin{tabular}{|c|c|}
\hline Parameter & Description \\
\hline $\mathrm{H}, \mathrm{h}_{1}, \mathrm{~h}_{2}$ & $\begin{array}{c}\text { Height of building, Tx and } \mathrm{Rx} \\
\text { antenna, respectively. }\end{array}$ \\
\hline $\mathrm{d}$ & $\begin{array}{c}\text { Unfolded distance } \\
\text { of a 2D path. }\end{array}$ \\
\hline $\mathrm{k}$ & $\begin{array}{c}\text { Index of each 3D path derived } \\
\text { from a 2D path on vertical section } \\
\text { plane. }\end{array}$ \\
\hline$\theta_{k}$ & $\begin{array}{c}\text { The elevation angle of the path } \\
\text { with respect to horizontal plane. }\end{array}$ \\
\hline$\Gamma\left(\theta_{k}\right), \tau\left(\theta_{k}\right), \mathrm{I}\left(\theta_{k}\right)$ & $\begin{array}{c}\text { Reflection, transmission } \\
\text { coefficient } \\
\text { and interaction coefficient. }\end{array}$ \\
\hline $\mathrm{n}$ & $\begin{array}{c}\text { The number of interactions in } \\
\text { 2D path. }\end{array}$ \\
\hline
\end{tabular}

Table 1. The parameters used in 3D wave propagation model

For each 3D path generated from a 2D path, the elevation angle of $k$-th ray radiated from the transmitter is computed by the following equation (2).

$$
\begin{gathered}
\theta_{k}=\arctan \left\{\frac{2 H\left[\frac{k+1}{2}\right]+(-1)^{k} h_{2}-h_{1}}{d}\right\} \\
\text { where } k \in\{1,2,3,4,5,6,7\} .
\end{gathered}
$$

Using the elevation angle computed as equation (2), the $3 \mathrm{D}$ propagation model is represented by the received power as given below.

$$
\begin{aligned}
& P^{3 D}(d)=\frac{P_{t} \lambda^{2}}{(4 \pi d)^{2}} \mid \sum_{k=-m}^{m} \cos \theta_{k} \sqrt{G_{T}\left(\phi_{T}, \theta_{k}\right) G_{R}\left(\phi_{R}, \theta_{k}\right)} \\
& \left.\times\left(\Gamma^{\text {ceiling }}\left(\theta_{k}\right)\right)\right)\left.^{\left[\frac{k+1}{2}\right]}\left(\left(\Gamma^{\text {floor }}\left(\theta_{k}\right)\right)\left[\frac{k}{2}\right]\right\rfloor \prod_{i=0}^{n} I_{i}\left(\theta_{k}\right)\right|^{2}
\end{aligned}
$$

\section{COMPARING 3D MODEL WITH 2D ONE}

The total received power predicted from the 3D model is believed to be more accurate than $2 \mathrm{D}$, because the $3 \mathrm{D}$ model counts more paths to predict the power which are not considered in 2D model. The values predicted from $3 \mathrm{D}$ model are not always larger than those using 2D. To see the performance of the simulation using each model, the simulation environment is depicted in Figure 7. The channel measurement is already performed in the same environment as used in the above simulations. $5.8 \mathrm{GHz}$ frequency signal are used in both measurement and simulation.

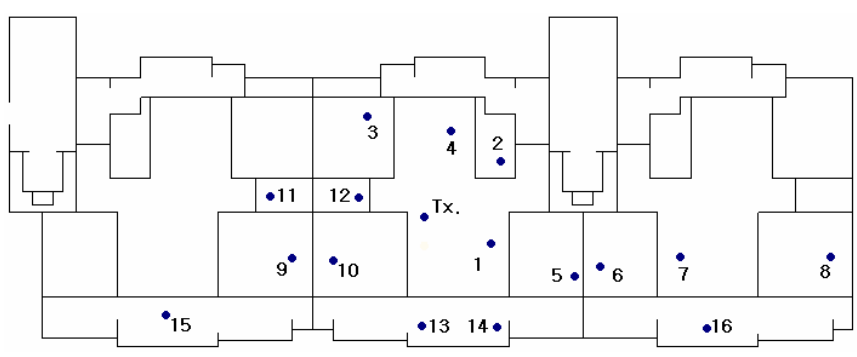

Figure 7. The indoor environment and the antenna location for measurements and simulations

The simulation results using 2D ray-tracing scheme and 3D one and measurement are shown in Figure 8.

Impulse Response (IR) includes almost all the information of the static PHY channel. Therefore, in order to compare the measured and simulated data, comparing the IRs obtained from 2D model, 3D model and the measured data at each receive point is reasonable work. To make the IR from the measurement data, we use the PN correlation method generally. However, the correlation value is often not a single value of one impulse but the complex sum of many copies in the form of isosceles triangles in small environments like indoor. That is, we can not recover the impulses neighboring within PN chip duration from the PN correlation profile in indoor environments. Thus the one to one comparison using IRs can not be realized easily. As the next best thing, we converted the deterministic IRs from the 2D and 3D simulations results into the form of PN correlation profiles like measured data form. The following graphs in the Figure 8 show the channel responses in the form of PN correlation at each receiving point.

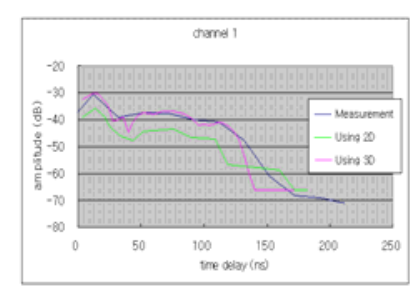

(1) at point 1

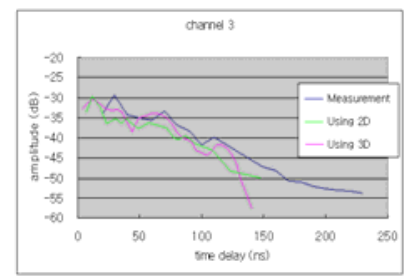

(3) IR at point 3

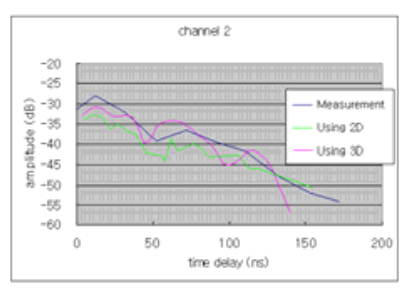

(2) at point 2

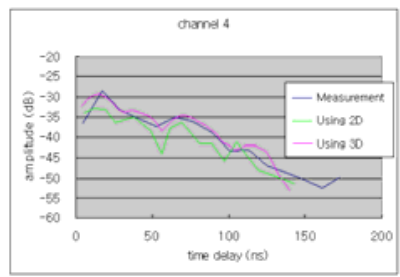

(4) IR at point 4 


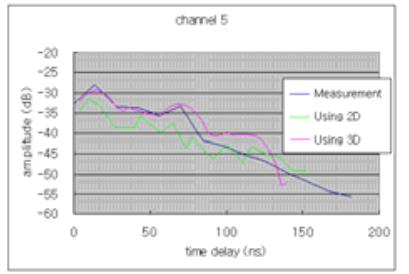

(5) IR at point 5

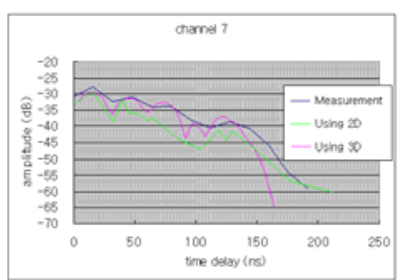

(7) IR at point 7

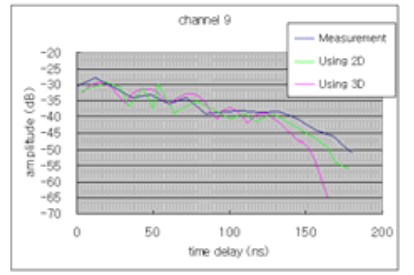

(9) IR at point 9

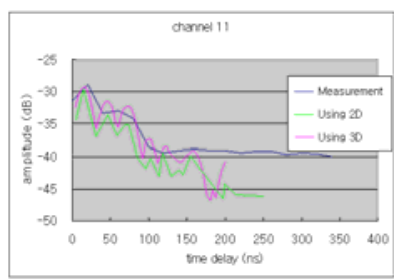

(11) IR at point 11

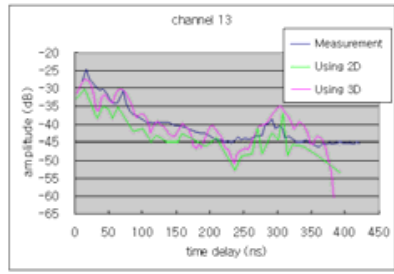

(13) IR at point 13

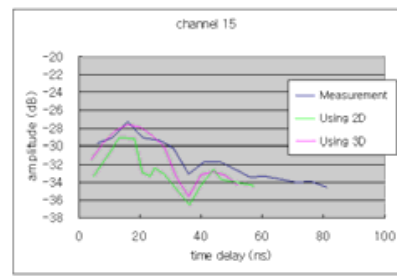

(15) IR at point 15

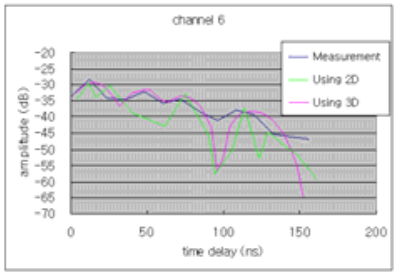

(6) IR at point 6

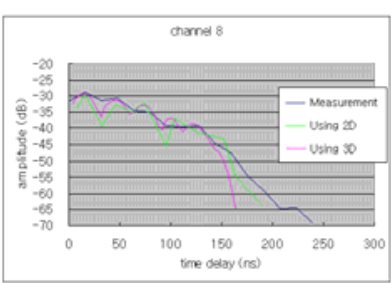

(8) IR at point 8

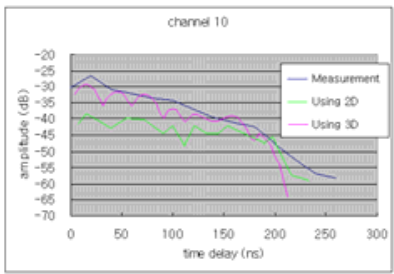

(10) IR at point 10

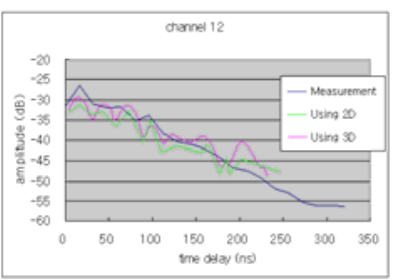

(12) IR at point 12

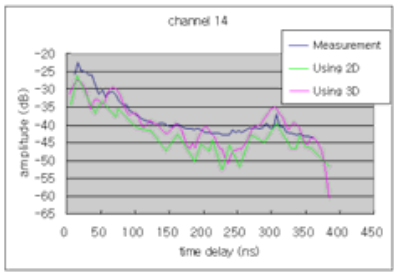

(14) IR at point 14

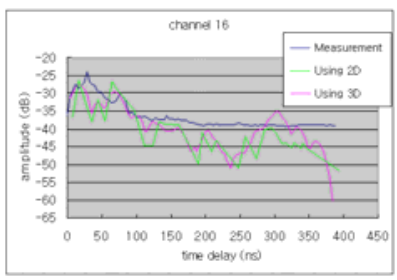

(16) IR at point 16
Figure 8. Channel responses using 2D, 3D simulation and channel measurement at each receiving point.

As shown in the above graphs, both results of simulations using 2D scheme and $3 \mathrm{D}$ one follow the over all trend of measured data roughly. However the measured power value is generally larger than the simulated one. This can be due to the effects of the scattering or the diffraction which is reckoned without, or due to the over-simplification of wave propagation model. The prediction error at each observation point is given in table 2 .

\begin{tabular}{|c|c|c|}
\hline \multirow{2}{*}{$\begin{array}{c}\text { Receiving } \\
\text { Point }\end{array}$} & \multicolumn{2}{|c|}{ Prediction error (dB) } \\
\cline { 2 - 3 } & Using 2D & Using 3D \\
\hline 1 & 7.4561 & 2.0105 \\
\hline 2 & 4.9275 & 2.4601 \\
\hline 3 & 5.2294 & 2.9325 \\
\hline 4 & 3.9178 & 0.9123 \\
\hline 5 & 6.4928 & 1.4567 \\
\hline 6 & 8.9427 & 4.4231 \\
\hline 7 & 7.8133 & 2.5019 \\
\hline 8 & 5.2348 & 1.9311 \\
\hline 9 & 1.6031 & 1.8949 \\
\hline 10 & 12.9202 & 3.129 \\
\hline 11 & 4.182 & 1.9364 \\
\hline 12 & 5.2991 & 2.8911 \\
\hline 13 & 5.8679 & 2.2855 \\
\hline 14 & 7.0236 & 3.2773 \\
\hline 15 & 3.4599 & 1.862 \\
\hline 16 & 9.1244 & 7.1485 \\
\hline
\end{tabular}

Table 2. comparison of prediction error between 2D and 3D raytracing scheme.

As shown in the table, the power prediction errors using 3D ray tracing are controlled within $5 \mathrm{~dB}$. This can be a remarkable improvement of prediction performance compared to $2 \mathrm{D}$ ray-tracing whose prediction error range is $5 \sim 10 \mathrm{~dB}$.

\section{CONCLUSIONS}

This paper presented the study about 2D and 3D raytracing scheme and their performance of channel prediction. Wave propagations at ceilings and floors which is considered in $3 \mathrm{D}$ ray-tracing technique are also studied and transmissions at ceiling and floor were proved to be negligible. 3D wave propagation model which is used for 3D ray-tracing simulation was made based on the analysis of 3D wave propagation phenomena. To see whether 3D ray-tracing scheme deserves to be applied, the comparison with 2D raytracing was performed and channel measurement data were used as a standard of comparison. In conclusion, $3 \mathrm{D}$ ray-tracing was proven to be a good tool for analyzing channel. And it can be used instead of channel measurement. 


\section{ACKNOWLEDGEMENT}

This paper was partly supported by ITRC, BK21 project

\section{REFERENCES}

[1] Joao Carlos Silva, Nuno Souto, A. Rodrigues, "Conversion of reference tapped delay line channel models to discrete time channel models,” IEEE VTC, vol. 1, pp 128-132, Oct. 2003

[2] V. Degli-Esposti, G. Lormbardi, C. Passerini, "Measurement and raytracing prediction of indoor channel parameters," Electronic Letters, vol. 34, pp 2167-2168, Oct. 1998.

[3] H. Suzuki, A. S. Mohan, "Measurement and prediction of high spatial resolution indoor radio channel characteristic map," Vehicular Technology, IEEE Trans., vol. 49, pp 1321-1333, July 2000.

[4] Athanasiadou. G.E, Nix A. R., “A novel 3-D indoor ray-tracing propagation model: the path generator and evaluation of narrow-band and wide-band predictions,” Vehicular Technology, IEEE Trans., vol. 49, pp 1152-1168, July 2000.

[5] Tarng, J.H., Chang, W.R., Hsu, B. J.,“Three-dimensional modeling of 900-MHz and 2.44-GHz radio propagation in corridors," Vehicular Technology, IEEE Trans., vol. 46, pp 519-527, May 1999.

[6] S. Y. Tan, M. Y. Tan, and H. S. Tan, "Multipath Delay Measurements and Modeling for Interfloor Wireless Communications," Vehicular Technology, IEEE Trans., vol. 49, pp 1334-1341, July 2000. 